ische Vorstellungen in den Reiseberichten europäischer Verfasser isoliert und deren in irgendeiner Weise strukturell relevant gewordene Reproduktion in der Physikalischen Geographie herausgearbeitet werden. Doch hier, wo die eigentliche philologische Arbeit beginnen müsste, lenkt Zhang bedauerlicherweise zur für ihre These vollkommen irrelevanten Frage nach Kants Rassismus über (vgl. 166).

Die an dieser Stelle formulierte Kritik am uneingelösten Versprechen einer nur formulierten, aber nicht im Detail nachgewiesenen Grundthese ändert freilich nichts daran, dass Zhang ein ungemein informatives und inspirierendes Buch geschrieben oder vielmehr aus den beinahe sämtlich bereits vorab publizierten Aufsätzen kompiliert - hat. Durchgängig werden die untersuchten Werke überzeugend in ihre historischen Kontexte und Diskurse eingeordnet, immer wieder beleuchtet Zhang instruktiv gewählte Textstellen unter ebenso überraschenden wie aufschlussreichen Gesichtspunkten: Forsters Vermittlung zwischen fremdartigem Eindruck und europäischen Denkmustern, Chamissos engagiertes Plädoyer für die wissenschaftliche Qualifikation seines
Freundes Kadu, Campes aufklärerische Kritik der Empfindsamkeit und sein präkolonialer Pragmatismus, Kotzebues in das Genre des Melodrams verpackte Gesellschaftskritik, Herders spannungsreicher Weg vom Relativismus zu einem universalen Historismus und Kants Betonung der Bedeutung geographischer Kenntnisse für das aufklärerische Projekt werden ebenso differenziert wie übersichtlich und nachvollziehbar dargestellt.

Dass ein roter Faden der ausführlichen Einleitung und den (allerdings äußerst knapp gehaltenen) Überleitungen zum Trotz am ehesten noch im letzten, offenbar eigens für die vorliegende Publikation verfassten Kapitel greifbar, ansonsten aber häufig vermisst wird, tut einer stets anregenden Lektüre keinen Abbruch im Gegenteil: Gerade der Verzicht auf eine lückenlose Einpassung aller untersuchten Elemente in das Gerüst einer einzigen Grundthese ermöglicht die vielen ungewöhnlichen Perspektiven, die Zhangs Abhandlung zu einer wirklichen Bereicherung der Forster-, Chamisso-, Campe-, Kotzebue-, Herder- und Kant-Forschung machen.

Heiko Ullrich

\title{
Benoît Ellerbach: L'Arabie contée aux Allemands: Fictions interculturelles chez Rafik Schami
}

Würzburg: Königshausen \& Neumann 2018 - ISBN 978-3826064845 - 49,80€

In seinem 2017 erschienenen Selbstporträt Ich wollte nur Geschichten erzählen: Mosaik der Fremde stellt der aus Syrien stammende Schriftsteller und Erzähler Rafik Schami nüchtern fest: »Ohne mein Exil in Deutschland gäbe es meine Romane und Geschich- ten nicht « (Schami 2017: 162). Zweifelsohne zählt Schami heute (nicht zuletzt auch aufgrund des jüngsten Kapitels syrischer Einwanderung nach Deutschland) zu den bekanntesten Stimmen einer inzwischen bereits über vierzig Jahre währenden Traditi- 
on deutschsprachiger Literatur mit Migrationskontext. Nun legt Benoît Ellerbach die erste umfassende Monografie zum >Phänomen Schami< vor, für die er einen literatursoziologischen Ansatz wählt, um sich auf die strategischen Positionierungen des Autors im literarischen Feld zu konzentrieren. Mit Blick auf die Prominenz Schamis - seine repräsentative Karriere, seine Produktivität und Präsenz in der Öffentlichkeit - erscheint eine solche Studie überfällig. Dass sie nun in französischer Sprache vorliegt, trägt sicherlich zum wissenschaftlichen Austausch zwischen zwei bedeutenden Literaturräumen mit Migrationserfahrung bei und fördert die Rezeption Rafik Schamis in Frankreich, wo gerade im letzten Jahr die Übersetzung von Sami und der Wunsch nach Freiheit beim Kinder- und Jugendbuchverlag >L'école des loisirs < erschienen und das Interesse an interkultureller Literatur grundsätzlich hoch ist. Ellerbachs Darstellung des Werdegangs des vielseitigen Autors, der 1946 in Damaskus geboren wurde, 1971 nach Deutschland kam und seit den 1980er Jahren Bücher in deutscher Sprache veröffentlicht, bietet zugleich einen Aufriss der Rezeptionsgeschichte von Migrationsliteraturen in Deutschland. Das ist auch insofern relevant, als Überlegungen zu Folgen von Einwanderung und zu transkulturellen Entwicklungen im deutschsprachigen Raum weiterhin hochaktuell sind. Dabei scheint Schami - obwohl omnipräsent in den Medien, wenn derzeit Stellungnahmen zum Konflikt in Syrien gefragt sind als Literat in der jüngeren Forschung etwas aus dem Blick geraten zu sein. Dies könnte daran liegen, dass seine Position innerhalb der deutschen Li- teratur, so etabliert und repräsentativ sie auch erscheinen mag, als einigermaßen singulär wahrgenommen wird. Aber wenn es darum ginge, einen Kanon deutschsprachiger Autor*innen zu definieren, die Deutsch als Fremdsprache erlernt haben, so gehört der zweifache Chamisso-Preisträger Schami fraglos dazu. Dies veranschaulicht Ellerbach insbesondere durch seinen Fokus auf die Wechselwirkungen zwischen Produktionsstrategien und Rezeption eines Werkes, das durch die Selbstinszenierung des Autors als Erzähler >in orientalischer Tradition eine höchst erfolgreiche Nischenposition eingenommen hat.

Für die Makroanalyse im ersten der beiden Teile des Bandes (Postures de l'auteur interculturel) und die Einordnung von Schamis Werk in den deutschen Literaturbetrieb verwendet Ellerbach das von Jérôme Meizoz entwickelte Konzept der posture auctoriale, mit dem die aktive Positionierung und agency von Autor*innen in Interaktion mit den Instanzen literarischer Rezeption beschreibbar wird. Der posture-Begriff umfasst sowohl die rhetorisch-diskursive als auch die soziologische Komponente von Autorschaft und vermag damit die Konstruktion des Autorethos in den Blick zu nehmen. Als besonders ergiebig erweist sich die Untersuchung von Schamis Positionierung im spezifischen Migrationskontext: Sei es die posture des politischen Engagements in der Anfangszeit seiner schriftstellerischen und herausgeberischen Tätigkeit im Umfeld der sogenannten Gastarbeiterliteratur; sei es die Rolle des unterhaltsamen Performers einer als >orientalisch in Szene gesetzten mündlichen Erzählwelt (Kritiker*innen titulierten 
ihn daraufhin wenig wohlmeinend als >Märchenonkel<) - oder schließlich die Position des Exilautors als Verfasser episch ausgreifender Geschichts- und Gesellschaftsromane, die das Lesepublikum seit den 20ooern in eine komplexe syrische Handlungs- und Erlebniswelt einführen (so in Die dunkle Seite der Liebe, Das Geheimnis des Kalligraphen und zuletzt in dem zeitgleich mit Ellerbachs Studie erschienenen Kriminalroman Die geheime Mission des Kardinals).

Die gut dokumentierte Produktions- und Rezeptionsanalyse leidet allerdings trotz des schlüssig hergeleiteten methodischen Ansatzes etwas darunter, dass die theoretische Prämisse der Marginalisierung hinter den aktuellen Forschungsstand zu transkulturellem Schreiben zurückfällt. So überrascht die eingangs formulierte Aussage über die geringe Kanonisierung eingewanderter Autor*innen: »[L]a littérature écrite aux XXème et XXIème siècles dans des pays germanophones par des auteurs allochtones peine toujours à se faire une place dans le canon littéraire allemand « (19). ${ }^{1}$ Eigentlich schon mit der Vergabe des IngeborgBachmann-Preises 1991 an Emine Sevgi Özdamar, spätestens aber seit der Auszeichnung von Melinda Nadj Abonji mit dem Deutschen Buchpreis im Jahr 2010 scheint eine solche Annahme widerlegt. Die Laureat*innen des Büchner-Preises 2018 (Terézia

1 | "Literatur von Autoren nichtdeutscher Herkunft, die im 20. und 21. Jahrhundert in deutschsprachigen Ländern entstanden ist, hat noch immer Mühe, einen Platz im deutschen Literaturkanon zu finden." (Alle Übersetzungen der Zitate aus dem besprochenen Band von M.G.)
Mora) und des Deutschen Buchpreises 2019 (Saša Stanišić) bestätigen die kontinuierliche Aufmerksamkeit für transkulturelles Schreiben, die sich auch über die Geschichte des häufig als Nischenförderung wahrgenommenen und inzwischen wieder eingestellten Adelbert-von-Chamisso-Preises hinaus fortsetzt. Ellerbachs Feststellung der offensichtlichen Verankerung von Schamis Werk in der deutschen Literaturlandschaft dank literarischer Auszeichnungen seit den 1990er Jahren (vgl. 39) relativiert dann auch die einleitend postulierten Exklusionstendenzen.

Die Mikroanalyse des zweiten Teils der Arbeit (Centre et périphérie dans les fictions interculturelles de Rafik Schami) bietet eine detaillierte narratologische Werkuntersuchung in chronologischer Reihenfolge, die sich im Wesentlichen auf Ansätze des französischen Poststrukturalismus (Deleuze/Guattari, Foucault) und postkolonialer Theorie (Said, Bhabha) stützt. Die für eine kritische Bewertung von Schamis posture relevante Analyse von Kanonisierungsprozessen führt bei der abschließenden Einschätzung allerdings zum Rückgriff auf nationalliterarische Kategorien, wodurch die dynamische Entwicklung des literarischen Felds der vergangenen dreißig Jahre gewissermaßen ausgeblendet wird:

En effet, on est en droit de se demander si Schami, en se tournant vers des thématiques et des problématiques en apparence déconnectées des réalités $\mathrm{du}$ monde germanique, ne cherche pas à faire de la langue allemande une langue cosmopolite [...], c'est-àdire une langue qui serait le véhicule 
d'une littérature dépassant les frontières des littératures nationales (439). ${ }^{2}$

Schamis interkultureller Ansatz scheint indessen von Beginn an einer nationalen Konzeption literarischer Produktion entgegenzustehen. Die insgesamt treffende Durchleuchtung seiner produktionsästhetischen Strategien hätte durch die Berücksichtigung wertvoller Erkenntnisse aus dem Bereich transnationaler und transkultureller Forschung (z.B. des Konzepts der postmonolingual condition, vgl. Yildiz 2012) sicher noch an Stringenz gewonnen. Offen bleibt am Ende, ob das Projekt einer »littérature arabe de l'exil [...] en conservant l'allemand comme langue d'écriture « ${ }^{3}$ (439) von Ellerbach als posture eines strategischen Exotismus oder als plausibler literarischer Ansatz bewertet wird. Diese insgesamt unentschiedene Haltung gegenüber Schamis Werk (dessen poetologischer Impetus paradoxerweise trotz einer Fülle von Hinweisen auf narrative Verfahren kaum erwogen wird) zeigt sich insbesondere in der interessanten Quellenstudie zu Schamis Märchenband Malula. Märchen und Märchenhaftes aus meinem Dorf (1987) im dritten Kapitel, der wichtige Über-

2 | "In der Tat darf man sich fragen, ob Schami durch die Zuwendung zu Themen und Fragestellungen, die dem Anschein nach keinen Bezug zu den Realitäten des deutschsprachigen Kulturraums haben, nicht die Absicht verfolgt, die deutsche Sprache zu einer kosmopolitischen Sprache zu machen [...], d.h. zum Medium einer Literatur, die die Grenzen von Nationalliteraturen überschreitet."

3 | [A]rabische Exilliteratur [...], wobei das Deutsche als Literatursprache beibehalten wird" legungen zum Einfluss von Walter Benjamins nostalgischem >Erzähler <Begriff auf Schamis narratives Projekt der réécriture vorangestellt werden. Ellerbach legt hier sehr überzeugend dar, in welcher Weise der Schriftsteller Aufzeichnungen und Textsammlungen deutschsprachiger Orientalisten aus dem 19. Jahrhundert mithilfe von Gotthelf Bergsträßers 1915 erstellter Buchausgabe Neuaramäische Märchen und andere Texte aus Malula verwendet hat, um auf der Basis dieses Materials zu einer eigenen literarisierten Adaptation der Texte zu gelangen, in denen das aramäische Substrat abermals für eine deutsche Rezeption aufbereitet wird. Die poetische Qualität dieses vielschichtigen Akts transkulturellen und transhistorischen Schreibens, mit dem palimpsestartigen Verschwinden der authentischen Quelle im Prozess des Erzählens (insbesondere durch die zweifache Aneignung und Veränderung in der deutschen Sprache), bleibt allerdings unerwähnt. Vielmehr wird das Verfahren als eine geschickte Form von Wiederaufbereitung dargestellt, bei der marktorientierte Faktoren poetischen Erwägungen mindestens gleichgestellt sind: »Car Schami semble y avoir découvert la possibilité de réécrire autrui tout autant que celle de se réécrire lui-même, dans une démarche qui se situe toujours à la frontière entre poétique et mercantilisme« (247). ${ }^{4}$ Unbestritten ist wohl, dass der Autor Schami be-

4 | "Denn Schami scheint darin die Möglichkeit der réécriture entdeckt zu haben, sowohl in Bezug auf andere als auch auf sich selbst, und zwar immer an der Grenze zwischen einem poetischen und einem marktorientierten Ansatz." 
stimmte Publikumserwartungen sehr erfolgreich erfüllt und sich damit ganz bewusst im Literaturbetrieb positioniert, dessen Mechanismen er aus seiner Perspektive des >Exoten < heraus von Beginn an kritisch beobachtet hat. Fraglich ist, ob das Streben nach Anerkennung und literarischem Erfolg unweigerlich mit »merkantilen Interessen« gleichzusetzen ist (wie Ellerbach in seiner Monografie suggeriert).

Ein spannender Ausblick der Studie sind Überlegungen zu einer gewissen >Verwandtschaft $<$ der beobachteten nomadischen und rhizomatischen Eigenschaften von Schamis Werk mit postnationalem und postkolonialem Schreiben in anderen Sprachen (genannt werden etwa Salman Rushdie, Amin Maalouf, Gabriel García Márquez). Tatsächlich geht Transkulturalität bei Schami wohl über die selbstdefinierte Rolle des Kulturmittlers zwischen Orient und Okzident und den interkulturellen Dialog hinaus. Dies zeigt nicht zuletzt der von Ellerbach ausgiebig analysierte Einsatz der Biografie zur fiktionalen Inszenierung einer narrativen Identität (vgl. Kapitel II La biographie comme moteur absolu de la narration). Es ist bedauerlich, dass die Untersuchung hier nicht auf aktuelle Forschungsergebnisse zurückgreift, die insbesondere das spielerisch-kreative Potential autofiktionalen Schreibens hervorheben (vgl. etwa Wagner-
Egelhaaf 2013). Schamis Ansatz auktorialer Rollenperformanz und narrativer Selbstdarstellung ließe sich aus dieser Perspektive heraus zweifellos noch differenzierter beleuchten. Zumal es ein typisches Merkmal der Migrationssituation ist, die eigene $>$ Fremdheit $<$ permanent (para-)literarisch zu reflektieren und daraus eine poetische Positionierung $\mathrm{zu}$ entwickeln. Ein Blick auf auto- und metafiktionale Strategien im Werk von Autor*innen mit vergleichbaren Biografien (z.B. Emine Sevgi Özdamar, Yoko Tawada, Abbas Khider, Saša Stanišić) hätte es ermöglicht, Schamis posture innerhalb einer spezifischen literarischen Tradition zu betrachten. Im Kontext von Migration und Exil führt das Spiel mit Identitäten und Verortungen unweigerlich $\mathrm{zu}$ einer amplifizierten Wirkung, da die Frage der Sichtbarkeit und Anerkennung im Literaturbetrieb unmittelbar damit verbunden ist. Durch seine präzise recherchierte und kritische Untersuchung von Schamis inszenierter Alterität legt Ellerbach auch die Komplexität der Rezeption migrantischen Schreibens offen. Nicht alle Analyseansätze vermögen gleichermaßen $\mathrm{zu}$ überzeugen, aber die Lektüre regt in jedem Fall zur weiteren Beschäftigung mit diesem so populären wie produktiven Schriftsteller an.

\section{Myriam Geiser}

\section{Literatur}

Schami, Rafik (2017): Ich wollte nur Geschichten erzählen: Mosaik der Fremde. Berlin/Tübingen.

Wagner-Egelhaaf, Martina (Hg.; 2013): Auto(r)fiktion. Literarische Verfahren der Selbstkonstitution. Bielefeld.

Yildiz, Yasemin (2012): Beyond the Mother Tongue: The Postmonolingual Condition. New York. 
